\title{
Adapting the Consumption of Carbohydrates for Diabetic Athletes
}

\author{
Anna Novials and Serafín Murillo \\ Additional information is available at the end of the chapter
}

http://dx.doi.org/10.5772/51570

\section{Introduction}

Nutritional recommendations associated with physical exercise performed by people with type 1 diabetes have changed in recent years, alongside the development of new drug therapies and discoveries in the field of nutrition. Before insulin was discovered in 1921, exercise was considered a dangerous activity, usually discouraged due to the high risk of metabolic disorder involved. Currently, exercise is not only considered safe but is prescribed as a basic treatment of the disease, essential for a healthy lifestyle and recommended for all patients with diabetes. The beneficial effects of regular physical exercise associated with diabetes are numerous, offering improved physical capacity, decreased cardiovascular risk and increased emotional and social wellbeing [1]. However, the specific role of exercise in improving glycemic control is currently under debate. The root of this debate lines in findings that show that the successful treatment of type 1 diabetes consists of creating and maintaining a balance among many different factors involved in glycemic control (kind of exercise, insulin dosage and diet). Therefore, the failure to adapt carbohydrate intake to insulin dosage or the characteristics of the physical activity to be performed may lead to significant glycemic imbalances.

In this sense, exercise-induced hypoglycemia is described as one of the main factors limiting physical exercise, as well as a cause of poor performance in athletes with type 1 diabetes. However, certain strategies, such as supplementing carbohydrate intake, can reduce the severity and duration of these episodes, thereby eliminating the fear of exercise-induced hypoglycemia [2].

The role of carbohydrates in the diet of athletes with diabetes is important as a prerequisite for achieving maximum athletic performance, not only in improving and accelerating the recovery of energy stores in the body, but also in regulating blood glucose levels during training sessions and competitions [3]. In general, the nutritional strategies used by athletes 
with and without diabetes should not differ. However, certain standards and guidelines must be established for including carbohydrate supplements within the set of specific treatments for diabetes.

\section{Carbohydrates and glycemic control}

Carbohydrates ingested in food are the main determinant of blood glucose levels following meals. Both the quantity and quality (or kind of carbohydrate) is particularly influential in the variability of blood glucose levels obtained after intake. One must bear in mind that foods rich in carbohydrates represent the basis of nutrition in most cultures, as they are important sources of vitamins, minerals and fiber [4].

Regarding the amount of carbohydrates, the minimum daily requirements are $130 \mathrm{~g}$ [5], with no studies available on the safety of nutritional guidelines calling for fewer carbohydrates during periods longer than one year. The quality or kind of carbohydrate ingested can cause significant variations in one's response to food, increasing or decreasing the rate at which the carbohydrates contained can be digested as well as their ability to raise blood sugar levels. Certain food-specific factors can increase the rate at which blood glucose levels rise, such as:

- Thermal or mechanical processing. Longer cooking times or certain mechanical processes, such as the milling of grain into flour, increase the food's absorption rate.

- Degree of starch gelatinization. Applying heat in the presence of water initiates the process of starch gelatinization, which facilitates the breakdown of food by intestinal enzymes during the digestive process, thereby increasing the absorption rate.

- Amylose-amylopectin ratio of starches. Starches are mainly made up of amylose and amylopectin chains. Amylose forms helical structures, unbranched, which are less accessible to digestive enzymes than amylopectin chains.

- Kind of sugar. Fructose has a slower absorption rate than glucose or sucrose. Furthermore, once absorbed by the intestine, it must undergo a series of processes by the liver in order to become glucose. Therefore, fructose-rich foods cause blood sugar to increase more slowly than those that contain other kinds of sugar, such as glucose or even starch, in their composition.

- Other food components. The presence of high amounts of protein, fiber or fat in food can alter the rate of absorption by slowing down the digestive process. Certain condiments such as vinegar are capable of acidifying food, which slows down the digestive process and therefore the absorption of food.

Furthermore, other factors specific to the individual must be taken into account that may also affect the rate of absorption. These factors include low blood glucose levels or fasting before eating. It should be noted that low carbohydrate intake during the hours or days prior to performing moderate or intense, mid- or long-duration physical activity can cause glycogen reserves in the liver and muscles to decrease, which then lowers the glycemic effect of any food eaten. 
One way to know the rate at which carbohydrates contained in a certain food increase blood sugar levels is by looking at its glycemic index. This measurement is made by comparing the blood glucose curve obtained after a quantity of food is ingested that provides $50 \mathrm{~g}$ of carbohydrates, as compared with the blood glucose curve obtained with a reference food, usually $50 \mathrm{~g}$ of glucose. Foods are grouped into three different categories, low, moderate and high, according to their glycemic index, with high glycemic index foods producing abrupt spikes in glycemic levels. Recently the concept of glycemic load has also surfaced, which is determined by taking into account the amount of carbohydrates contained in the portion of food consumed.

It is important to remember that measurements are a theoretical concept and that there is a wide range of factors affecting the rate at which carbohydrates are absorbed and raise glycemic levels. These factors include the kind of food, brand or country of origin, cooking methods used, and degree of food processing.

At any rate, in diabetes management it is important to coordinate insulin doses with the carbohydrate content of meals, which can be estimated using a variety of methods including carbohydrate counting, the carbohydrate exchange system, or experience-based serving size approximations.

\section{Effect of exercise on blood glucose balance and the risk of hypoglycemia}

The effect of exercise on blood glucose levels is determined by the interaction that takes place between metabolic and hormonal effects, as well as changes produced in muscle glucose uptake.

\subsection{Energy substrates during rest and exercise}

Fat deposits of adipose tissue are the major source of energy in the human body, with a reserve of between 60,000 and 150,000 Kcal. This content is much greater than the energy that carbohydrates can provide, approximately 2,000 Kcal. Most of these, about $1500 \mathrm{kcal}$, are stored as glycogen in the muscles, and the rest comes from glycogen deposits accumulated in the liver and from glucose found in the blood and extracellular fluids.

During the early stages of exercise [6-8], muscle glycogen is the main energy source for muscle contraction. Subsequently, the depletion of muscle glycogen deposits activates the lipolysis of fatty acids stored in adipose tissue. Thus, the increase of free fatty acids and glycerol in plasma constitutes an additional energy source for muscle contraction. Glucose will then be provided by hepatic glycogenolysis followed by hepatic gluconeogenesis. The substrates used by the liver to synthesize new glucose are lactate, pyruvate and certain amino acids, mainly alanine, together with the glycerol derived from the metabolism of triglycerides. The contribution of different substrates depends on the intensity, duration and kind of exercise performed. The relative proportion of each seeks to maintain three fundamental physiological aspects: 1) to preserve blood glucose 
balance, 2) to maintain the efficient metabolism and storage of glucose, and 3) to preserve and maintain deposits of muscle glycogen to avoid and/or delay the onset of muscular fatigue.

\subsection{Hormonal response to exercise}

Metabolic adjustments to exercise are possible thanks to a highly efficient system that integrates nerve impulses and hormonal response. During rest, individuals without diabetes experience what is called a basal insulin secretion, which increases in response to the rise in blood glucose that occurs after ingesting food. It is well known that insulin stimulates glucose uptake by skeletal muscle and by the liver, to later facilitate its storage in the form of glycogen.

Exercise causes adrenergic nerve stimulation, acting on pancreatic beta-cells and leading to inhibited insulin secretion [9-10]. Fortunately, this decrease in insulin levels does not affect muscle glucose uptake, as exercise triggers other mechanisms that can improve muscle glucose uptake.

Stimulation of another pancreatic hormone, glucagon, and, in particular, its close and proper interaction with insulin, are phenomena taking place during the exercise that are essential to maintaining and regulating glucose production. Decreased insulin levels at baseline exercise promote increased glucagon secretion by alpha cells in pancreatic islets. This increase is critical and acts directly on the metabolic pathways of hepatic glucose production (glycogenolysis and gluconeogenesis). Also, during exercise, counter-regulatory hormones increase, such as catecholamines, cortisol and growth hormone, promoting the balance of the aforementioned metabolic pathways and increasing lipolysis in fat cells. High-intensity exercise can activate these counterregulatory hormones in an exaggerated way, resulting in a noticeable increase in hepatic glucose production and a moderate degree of hyperglycemia when exercise is over.

\subsection{Metabolic responses to exercise in diabetes}

As expected, athletes with type 1 diabetes are different from their non-diabetic counterparts, as they have an insulin secretion deficiency and, consequently, their counterregulatory hormones respond differently. Patients with diabetes treated with insulin should learn to mimic their own natural insulin secretory rhythm, in response to physiological changes induced by exercise.

Any patient with diabetes and, especially, diabetic athletes should prevent problems arising from poor insulin dosage. If an athlete with diabetes starts exercise with a significant insulin deficiency, his/her response to exercise may trigger a hyperglycemic overcompensation, even ketosis, since a lack of insulin induces: 1) increased hepatic glucose production; 2) decreased peripheral glucose utilization; and 3) excessive lipolysis with an increase in free fatty acid production. 
If an athlete with diabetes, under any circumstances, experiences an excess of insulin when exercising, his/her response to it can trigger hypoglycaemia, due to: 1) decreased hepatic glucose production; 2) increased peripheral glucose utilization; and 3) reduced lipolysis [11-12].

It is also important to consider the changes that occur after physical activity. Muscle glucose uptake independent of insulin is still stimulated during the hours following activity, and muscles need more quantity of glucose to replenish glycogen stores. Exercise increases insulin sensitivity, which means that the effects of insulin last longer. If an athlete with diabetes not properly increase carbohydrate intake and/or reduce insulin dosage, a significant episode of hypoglycemia may occur hours after exercise has ended [13].

\subsection{Effects of insulin and exercise on muscle glucose uptake}

Both insulin and muscle contraction help glucose enter the muscles, where it is oxidized and subsequently turned into energy for muscle contraction. However, the mechanisms by which these two stimuli facilitate glucose transport are not entirely understood. There is evidence suggesting that they do not act in a similar manner. For example, exercise, unlike insulin, induces an increase in muscle blood flow and glucose transport, which persists for hours after exercise has ended. Experiments with laboratory animals have demonstrated that the induction of glucose transport produced by exercise is independent of insulin, as tyrosine kinase activity of the insulin receptor is not stimulated. Instead, it appears to act through other pathways, in particular, those involving the AMP kinase (AMPK).

Molecular characterization of glucose transporter GLUT 4, which is specifically expressed in muscle and fat cells, has shed new light on elucidating the mechanisms mentioned above. It appears that the translocation of GLUT 4 [14-15] from the cytosol to the cell membrane is one of the main mechanisms of glucose transport in muscles and can be stimulated both by insulin and by exercise. The existence of another type of transporter in muscle, GLUT 1, has also been demonstrated, although its function is not entirely known. The important role of certain enzymes such as hexokinase and glycogen synthase also highlights the complex system of glucose transport into muscles. AMPK [16] emerges as an important molecule regulating multiple metabolic processes that occur in skeletal muscle, in response to exercise. It appears that muscle contractions induced by exercise increase the activity of the enzyme, which, in turn, stimulates glucose transport.

\section{Factors that determine carbohydrate needs during and after physical exercise}

A prediction of one's consumption of carbohydrates during and in the hours after physical activity requires a great knowledge and analysis of factors specific to each type of activity. In this sense, the personal observations and experience of each athlete are highly important, assessing the effect of each type of activity on his/her blood sugar levels. It is recommended to monitor blood glucose levels before and after exercise, or, if possible, to use a continuous glucose sensor. 
In general, the characteristics of the activity to be performed must be known, as these factors affect blood glucose levels to a high degree:

- Type of exercise: Forms of exercise dominated by the aerobic component, such as walking, running, swimming, skating or cycling, produce greater glucose consumption and, therefore, have a stronger hypoglycaemic effect. In contrast, forms of exercise with an elevated anaerobic component, like sprints, combat sports and working with heavy weights, can produce strong adrenergic stimulation (stimulating liver production of glucose) and thus usually have a lesser hypoglycaemic effect post-exercise. Competitive sports can also be associated with significant emotional stress (high adrenergic stimulation), which causes increased blood sugar after exercise, especially in children and adolescents.

- Duration: During the first 30-60 minutes of moderate- or high-intensity exercise, muscular and hepatic glycogen becomes the primary muscle fuel. Thereafter, the glycogen stores begin to decrease, and muscles increasingly obtain energy from fatty acids and plasmatic glucose. Following this is when the most significant changes in blood glucose are observed.

- Intensity: Glucose is the preferred muscle fuel for exercise performed at a moderate or high intensity, whereas low-intensity exercise uses fatty acids as energy source. Therefore, low-intensity activities such as walking may have a minimal effect on blood sugar, whereas intense activities such as running could cause a stronger and faster blood glucose lowering effect.

- Frequency: Hypoglycaemic effects, especially after exercise, increase after several consecutive days of physical exercise. In this situation, since it is virtually impossible to recover of glycogen stores from one day to another, the body is less able to use the mechanisms of hepatic glycogenolysis to regulate low blood glucose.

- Schedule: The timetable of insulin administration results in the presence of different blood insulin levels throughout the day, which brings about a greater tendency to develop hypoglycemia during physical activity. This effect is more likely when exercise is performed just within 2-3 hours after meals, when rapid-acting insulin acts the strongest.

It is important to keep in mind that an athlete's level of fitness also affects his/her glucose uptake. For this reason, athletes just starting a particular sport usually consume more glucose than those who are more advanced in the sport, as training helps the body to use fat as muscle fuel, preserving most glucose reserves as glycogen.

\section{Recommendations for carbohydrate supplementation in diabetic athletes}

Nutritional guidelines for athletes with diabetes are similar to those for all athletes, in accordance with the activity performed. Thus, the recommended total amounts of macro and micronutrients should be calculated taking into account the characteristics of each individual as well as the specific requirements of the different stages of training or 
competition. However, in the case of carbohydrates, in addition to overall quantitative control, the distribution of these carbohydrates throughout the day takes on even more importance, according to the exercise habits and insulin dosage followed by each athlete.

Carbohydrates represent the main nutrient for both ensuring optimal muscle work and for maintaining blood glucose levels as close to normal as possible. Since reserves of carbohydrates in the body are limited, nutritional strategies must aim at maintaining or quickly replenishing these deposits once consumed through physical exercise. In this sense, dietary guidelines that heavily restrict carbohydrates are not recommended, as they would decrease athletic performance and at the same time increase the risk of hypoglycemia.

The kind of carbohydrates recommended depends on the timing of when they are ingested in relation to when exercise is performed. Thus, it is possible to use slow-absorbing carbohydrates (low-glycemic index) in the hours prior to a workout or competition, but also fast-absorbing ones (high-glycemic index) just before or during the activity. Also, continous blood glucose measurements should be used to monitor the rate at which the body needs glucose, bearing in mind the type of food most appropriate for each situation.

On another front, studies in people without diabetes found a better recovery of glycogen stores after exercise when high glycemic index foods were taken [17]. In contrast, in athletes with type 1 diabetes, priority was given to the total amount of carbohydrates over the kind of carbohydrates ingested [18]. Further studies are needed to assess the importance of following nutritional strategies based on the use of high glycemic index foods in the postexercise recovery of athletes with type 1 diabetes.

\subsection{Dietary planning}

Carbohydrate intake should be adapted to the needs of each given moment, in accordance with each phase of general conditioning or training for competitions.

\subsubsection{Diet during the training phase}

In daily training, an athlete's diet must maintain a high percentage of carbohydrates, in order to replenish the muscle and liver glycogen that has been spent. Conserving glycogen stores helps reduce the frequency of hypoglycemia during and in the hours following exercise. It should be noted that hepatic glycogenolysis is one of the main mechanisms involved in restoring blood glucose levels during the process of hypoglycemia and that a lack of liver glycogen stores decisively limits normal functioning.

In individuals without diabetes who train for less than 1 hour a day, the required carbohydrate intake is about 5-6g of carbohydrates / kg of body weight per day, reaching 8$10 \mathrm{~g} / \mathrm{kg}$ weight / day if the training exceeds 2 hours a day. Unfortunately, there are no studies evaluating the effectiveness of these nutritional strategies in athletes with type 1 diabetes, under the assumption that they should be any different for athletes with type 1 diabetes who maintain the disease under optimum control. 


\subsubsection{Diet during the pre-competition phase}

Many athletes have adopted the strategy of carbohydrate loading during the week prior to a running or cycling competition. In general, carbohydrate loading is not recommended, especially when performed after 2-3 days of carbohydrate restriction. This strategy, used by some athletes to increase muscle glycogen stores, puts athletes with diabetes at risk, especially during the period of carbohydrate restriction, leading to even metabolic imbalance and ketosis. On the other hand, ingesting very high amounts of carbohydrates during the loading period can provoke blood sugar fluctuations that could even decrease the athlete's performance during competition. This kind of dietary requires increasing the frequency of capillary blood glucose measurements in order to maintain glycemia within normal range.

At any rate, on the days prior to a competition, a high-carbohydrate diet is recommended, near or, if possible, above the guideline of $8 \mathrm{~g} / \mathrm{kg}$ weight/day.

\section{Nutritional composition of foods commonly used in sports}

During exercise it is advisable to increase carbohydrate intake, of both low and high glycemic index values. However, glycemic levels must be considered before recommending a certain food or drink or another. Below is a description of the carbohydrate content of some of the foods and drinks most commonly used while performing physical exercise:

- Isotonic drinks: These contain a sugar content of 5 to $8 \%$ (between 5 and $8 \mathrm{~g}$ for every $100 \mathrm{cc}$ ), the concentration that is most easily digested, allowing for a good tolerance of the drink while practicing sports. Moreover, they provide sodium, potassium or chlorine, electrolytes that help replenish lost minerals, thus, they are especially appropriate for activities lasting 1 hour or longer.

- Soft drinks: This group includes cola and orange soft drinks, tonic water, and carbonated drinks containing sugar. Their sugar content is around $10 \%$, which makes their digestion more difficult when exercise is high-intensity. Furthermore, cola soft drinks contain caffeine, a substance which increases dehydration during exercise.

- Energy drinks: These drinks have a high sugar content (above 10\%) and, in addition, contain substances to which anti-fatigue properties are attributed, such as 2aminoethanesulfonic acid or ginseng. Their high sugar content and stimulating effects do not make them recommendable as a supplement during physical exercise.

- Fruit juices: The distinction between natural and commercial fruit juices must be made. Natural juices have a relatively low carbohydrate content (4-6\%), allowing them to be used in a way similar to isotonic drinks. This also applies to commercial juices with "no added sugar", as they contain only the sugar naturally found in fruit (fructose) without adding any other kind of sugar later during processing. In contrast, commercial fruit juices with added sugar during processing have a total sugar content of around $10 \%$ (similar to that of soft drinks), in which case consumers are advised to read nutrition labels, as manufacturers add different quantities of sugar. 
Another difference between the two is that natural juices raise glycemic levels much more slowly than commercial juices, as they consist primarily of fructose.

- Glucose tablets: Pure glucose is the substance that raises glycemic levels most quickly. Occasionally it can produce some digestive discomfort, such as abdominal pain or diarrhea, produced when large quantities of glucose enter the intestine. Tablets are recommended to be taken little by little and always with water, in order to facilitate their absorption.

- Glucose gels: These consist of a mixture of glucose (or other forms of sugar) with water and fruit flavorings, forming a textured emulsion similar to that of honey or marmalade, more pleasing than that of glucose tablets or pills. However, they can also produce some digestive discomfort in certain individuals, so they are also recommended to be taken in small servings and always with abundant liquids.

- Energy bars: These are made from a grain or flour base, with a certain amount of added sugar or protein. Energy bars serve two different purposes during exercise: in addition to maintaining glycemic levels, they also help ward off hunger during long-duration exercise. They are very practical, as they are easy to transport and store and also easy to digest, rarely creating digestive discomfort.

In terms of their effects on glycemic levels, certain differences are found between energy bars, according to the ingredients used. Thus, grain bars increase glycemia faster than those with a higher protein content. There are also enery bars made with dried fruit as an alternative to traditional grain bars.

\begin{tabular}{|c|c|c|c|}
\hline Food/drink & Serving & $\begin{array}{c}\text { Carbohydrates (g) } \\
\text { per serving }\end{array}$ & Carbohydrates (\%) \\
\hline \multicolumn{4}{|l|}{ Isotonic drinks } \\
\hline Isostar $^{\circledR}$ & $200 \mathrm{ml}$ & 14 & 7 \\
\hline Gatorade $^{\circledR}$ & $200 \mathrm{ml}$ & 12 & 6 \\
\hline Aquarius $^{\circledR}$ & $200 \mathrm{ml}$ & 12 & 6 \\
\hline Powerade $^{\circledR}$ & $200 \mathrm{ml}$ & 13 & 6,5 \\
\hline \multicolumn{4}{|l|}{ Other drinks } \\
\hline Soft drinks & $200 \mathrm{ml}$ & 20 & 10 \\
\hline Commercial fruit juices & $200 \mathrm{ml}$ & 24 & 12 \\
\hline Redbull & $200 \mathrm{ml}$ & 22 & 11,2 \\
\hline \multicolumn{4}{|l|}{ Foods } \\
\hline Orange & Medium size, $130 \mathrm{~g}$ & 10 & 8 \\
\hline Apple & Medium size, $130 \mathrm{~g}$ & 12 & 9 \\
\hline Banana & Small size, $80 \mathrm{~g}$ & 16 & 20 \\
\hline Graham crackers & 3 units, $21 \mathrm{~g}$ & 13 & 63 \\
\hline Bread & 1 large slice, $30 \mathrm{~g}$ & 14 & 47 \\
\hline Glucose tablets & 2 units, $10 \mathrm{~g}$ & 10 & 99,5 \\
\hline Energy bars & 1 unit, $25 \mathrm{~g}$ & 15 & 60 \\
\hline
\end{tabular}

Table 1. Carbohydrate content of certain foods and drinks used during exercise 


\section{Carbohydrates in preparing for a competition}

\subsection{Nutrition during the hours prior to competition}

If exercise is performed right after fasting or an initial nutritional deficiency, the reserves of glycogen in the liver are reduced, which also leads to a premature decrease in glycemic levels, implicating a greater risk of hypoglycemia as well as a noticeable decrease in performance. Therefore, diabetic athletes must eat a meal rich in carbohydrates during the 3 hours prior to a training session or competition lasting 45-60 minutes or more. This meal must preferably include foods rich in low-glycemic index carbohydrates.

For short-duration exercise, pre-competition strategies are not as important, as the depletion of glycogen reserves is normally not as crucial to the overall performance of the athlete.

One strategy for controlling glycemic levels consists of measuring the levels of glucose in the blood 15-30 minutes before a competition and, depending on the levels obtained, taking a supplement of $15 \mathrm{~g}$ of high glycemic index carbohydrates, in order to prevent hypoglycemia during exercise. This supplement is omitted if blood glucose levels are above $150-200 \mathrm{mg} / \mathrm{dl}$ or higher, in accordance with the kind of exercise performed and the individual needs of each athlete. Including supplements of this nature has been found to reduce the frequency of hypoglycemia also during the hours following physical activity [19].

Before beginning any kind of exercise, the measurement of blood glucose levels is recommended. Thus, the intake of carbohydrates before physical activity begins is conditioned by the glycemic levels detected beforehand. On one hand, physical activity is not advised when levels are above $250 \mathrm{mg} / \mathrm{dl}$ and ketone bodies are present, or should be performed with caution when glycemic levels are above $300 \mathrm{mg} / \mathrm{dl}$. In this case, performing exercise could represent a risk, as physical activity itself increases the production of ketone bodies, which could considerably worsen the metabolic state of the athlete.

On the other hand, glycemic levels before exercise found to be lower than $100 \mathrm{mg} / \mathrm{dl}$ require the administration of high glycemic index carbohydrates in order to prevent hypoglycemia during the initial minutes of physical activity [21]. This extra supplement should provide 10 to 15 grams of carbohydrates.

\subsection{Nutrition during competition}

Before beginning mid- or long-duration exercise, reducing insulin doses prior to the activity may not be sufficient but also require an increased intake of carbohydrates during exercise. This is the case of mild- and long-duration exercise (more than 60-90 minutes) and, in particular, unplanned exercise sessions, reducing insulin doses beforehand is not possible.

These carbohydrate supplements should be individualized according to the previously described factors. Table 2 offers approximate target ranges that may prove useful for people with diabetes who are taking up a sport or physical activity. 


\begin{tabular}{|l|c|c|c||}
\hline $\begin{array}{l}\text { Exercise intensity } \\
\left(\% \mathbf{O O}_{2 \max }\right)\end{array}$ & $<20$ minutes & $\mathbf{2 0 - 6 0}$ minutes & $>60$ minutes \\
\hline $25 /$ light & $0-10 \mathrm{~g}$ & $10-20 \mathrm{~g}$ & $15-30 \mathrm{~g} / \mathrm{h}$ \\
\hline $50 /$ moderate & $10-20 \mathrm{~g}$ & $20-60 \mathrm{~g}$ & $20-100 \mathrm{~g} / \mathrm{h}$ \\
\hline $75 /$ intense & $0-30 \mathrm{~g}$ & $30-100 \mathrm{~g}$ & $30-100 \mathrm{~g} / \mathrm{h}$ \\
\hline
\end{tabular}

Table 2. Carbohydrate supplements according to intensity and duration of exercise

These recommendations must be adapted and tailored to the individual needs of each athlete and the doses of carbohydrates confirmed for each of the training sessions or competitions performed. To do so, glycemic levels should be monitored both before and after physical activity, searching for patterns particular to each athlete.

Unplanned physical exercise deserves special attention. In this situation, adjusting insulin doses may not be possible, and hypoglycemia must be prevented exclusively by an increased intake of carbohydrates. The time of day at which the activity is performed must also be considered; when under the maximum effect of a fast-acting insulin analog or hypoglycemic drug, hypoglycemia must be prevented by administering a greater amount of carbohydrates. An initial supplement of $10-30 \mathrm{~g}$ of carbohydrates should be taken, with continued carbohydrate intake depending on the intensity and duration of the exercise to be performed (see Table 2).

Diabetic athletes, in order to prevent hypoglycemia, often consume an excess of carbohydrates both before and after exercise. In other cases, some tend to overestimate the effects of physical activity on glucose consumption, with the belief that exercise alone will reduce any excesses in their diet. Special emphasis must be placed on adjusting carbohydrate intake to the expected duration and intensity of the exercise, as in some athletes who are very accustomed to a particular kind of exercise the reduced insulin doses and increased carbohydrate intake do not meet the recommendations in clinical guides [22].

\subsection{Nutrition after competition}

Once the activity is over, the replenishment of glycogen reserves and increased sensitivity to insulin continue for several hours, even until the day after the training session or competition.

Athletes with type 1 diabetes therefore must know that during this period they are more prone to suffer hypoglycemia. The optimal intake of carbohydrates consists of $1,5 \mathrm{~g}$ per $\mathrm{kg}$ of body weight. These carbohydrates should preferably be high glycemic index foods, to be taken during the first 30 minutes immediately following physical activity. Ideally, another dose of $1,5 \mathrm{~g}$ of carbohydrates per $\mathrm{kg}$ of body weight should be taken before 120 minutes have elapsed after the physical activity has ended. 
Although this amount of carbohydrates is the recommendation for replenishing glycogen reserves in the muscles and liver in all athletes, the habits of athletes without diabetes make it even more difficult for athletes with type 1 diabetes to achieve proper glycemic control. The recommended quantities may need to be accompanied by insulin in order to prevent hypoglycemia, therefore, nutritional strategies are recommended only for those diabetic athletes with significant experience in handling the disease. At any rate, the amount of carbohydrates in post-exercise supplements should be adjusted to the duration and intensity of the exercise performed, beginning very gradually and in association with a greater number of capillary glycemia measurements.

During the hours following exercise, glucose needs are increased, even 12-16 hours after physical activity has ended. This phenomenon is due in part to an increased sensitivity of muscle cells to glucose and in part to the need for replenishing glycogen reserves depleted during exercise.

As a result, this increase in glucose consumption following exercise raises the risk of suffering episodes of hypoglycemia, which must be prevented by changes in diet and in insulin doses. For this reason, once physical activity has concluded, it is advisable to check glycemic levels and evaluate the need to take food supplements containing carbohydrates, in accordance with the obtained results:

- Glycemia less than $120 \mathrm{mg} / \mathrm{dl}$ : take a supplement containing 15-20g of high glycemic index carbohydrates.

- Glycemia between 120 and $200 \mathrm{mg} / \mathrm{dl}$ : supplements are usually not required.

- Glycemia greater than $200 \mathrm{mg} / \mathrm{dl}$ : carbohydrate supplements are usually not required and the possibility of including a fast-acting insulin analog should be considered ${ }^{1}$.

Furthermore, in order to prevent post-exercise hypoglycemia, it may be necessary to compensate for the elevated consumption of glucose by the muscles through reducing insulin doses during the hours following exercise, both in fast-acting insulin as well as in slow-acting insulin for the basal needs of those using an insulin pump. The magnitude of this decrease in insulin depends mainly on the intensity and duration of the exercise performed, keeping in mind that the hypoglycemic effect of exercise is greater during the first 60-90 minutes afterwards [22].

\section{Adapting nutrition to different kinds of physical exercise}

The following are examples of nutritional strategies recommended for certain kinds of exercise:

- Short-duration, low-intensity exercise: Exercise such as walking, riding a bike or light swimming, for a period of time less than 30 minutes, is associated with low glucose consumption, thus, its hypoglycemic effect is small. Normally adjusting insulin treatment and increasing carbohydrate intake are not necessary.

\footnotetext{
${ }^{1}$ Athletes with diabetes must keep in mind that the effects of insulin following exercise are also much greater than normal.
} 
- Short-duration, high-intensity exercise: This group includes swimming races or track and field events, such as sprints and hurling (for example, 100 meter dash, shot-putting, etc.), and also combat sports like judo or taekwondo. The brief duration of the exercise means that the complete depletion of glucose is not as significant. Moreover, the elevated intensity of the exercise produces adrenergic activation, which increases the production of glucose by the liver. For these reasons, adjusting insulin doses and supplementing with carbohydrates are usually not necessary.

- Mid-duration aerobic exercise: Training for cycling, swimming or track events, as well as spinning, aerobics or step aerobics sessions, lasting approximately one hour, usually produce a more pronounced hypoglycemic effect. Nevertheless, since the sessions are still relatively brief, the decrease in insulin should be in the range of $10-20 \%$ prior to the activity, accompanied by an intake of $20-30 \mathrm{~g}$ of carbohydrates, preferably fastabsorbing.

- Long-duration exercise: Sporting events such as cycling competitions, triathlons or marathons involve performing exercise of a moderate or high intensity for prolonged periods of time. The consumption of glucose is quite high, depending on the intensity and duration of the activity. The risk of hypoglycemia is very high, both during the activity and up to 24 hours after the activity has ended.

In this case, adjustments in treatment are much more severe. A decrease in insulin of $25-50 \%$ is recommended prior to the onset of activity, which, in the case of long-duration competitions (more than 4 hours), could reach a $75 \%$ or greater reduction. The use of fastacting insulin following the activity should also be reduced, by about 10 to $20 \%$. In a similar fashion, the intake of carbohydrates should increase throughout the activity, according to the intensity of exercise involved.

- Team sports: In sports such as soccer, basketball or volleyball, the consumption of glucose can be quite variable, involving factors such as the position of the player on the team, the conditions of the game, and even the scoreboard.

Adjustments in treatment include reducing insulin doses prior to the activity by $10-20 \%$, along with the intake of $10-20 \mathrm{~g}$ of carbohydrates for every 30 minutes of activity.

\section{Conclusions}

A proper dietary education proves to be absolutely essential for athletes with type 1 diabetes to achieve optimal results. The objective of nutritional therapy is two-fold: on one hand, to maintain glycemic levels as normal as possible, and, on the other, to achieve the maximum sporting performance possible.

For this purpose, it is necessary to understand the functioning of the energy pathways of the body, as well as its hormonal response to exercise, with the goal of being able to foresee future glycemic response to the activity performed. 
Nutritional recommendations regarding the intake of carbohydrates geared toward athletes with type 1 diabetes should not be any different from those geared toward other athletes. Nevertheless, diabetic athletes must alter the way in which they incorporate carbohydrates into their normal diet, in order to produce the smallest changes possible in their glycemic levels. Thus, it is important to properly distribute these carbohydrates among the various meals of the day, keeping in mind the dosage of insulin to be used, while planning different training sessions or competitions.

Finally, dietary changes should take into account the characteristics of the physical activity, such as the kind of exercise, intensity and duration, as well as time of day at which it is performed.

\section{Author details}

Anna Novials and Serafín Murillo

Department of Endocrinology and Nutrition, Hospital Clínic de Barcelona, Barcelona, Spain

Institut d'Investigacions Biomèdiques August Pi i Sunyer (IDIBAPS), Barcelona, Spain

Spanish Biomedical Research Centre in Diabetes and Associated Metabolic Disorders (CIBERDEM), Spain

\section{Acknowledgement}

We thank K. Katte [from Spanish Biomedical Research Centre in Diabetes and Associated Metabolic Disorders (CIBERDEM)] for language consultancy.

\section{References}

[1] Schneider SH, Ruderman NB (1990) Exercise and NIDDM (Technical Review). Diabetes Care.13:785-789.

[2] Vrazeau AS, Rabasa-Lhoret R, Strychar\&Mircescu H (2008) Barriers to physical activity among patients with type 1 diabetes. Diabetes Care.31:2108-2109.

[3] Nagi D (2006) Exercise and Sport in Diabetes. Wiley \& Sons Ltd.Chichester. England.

[4] American Diabetes Association (2008) Position Statement: Nutritional recommendations and principles for individuals with diabetes mellitus. Diabetes Care. 31 (Suppl 1): S61-S78.

[5] Institute of Medicine. (2002) Dietary Reference Intakes: Energy, Carbohydrate, Fiber, Fat, Fatty Acids, Cholesterol, Protein, and Amino Acids. Washington, DC, National Academies Press.

[6] Wasserman DH (1995) Control of glucose fluxes during exercise in the postabsortive state. Annual Review of Physiology. Palo Alto, CA, Annual Reviews, p 191-195. 
[7] Zinker BA, Bracy D, Lacy DB, Jacobs J, Wasserman DH (1993) Regulation of glucose uptake and metabolism during exercise: an in vivo analysis. Diabetes.42: 956-965.

[8] Horowitz JF, Klein S (2000) Lipid metabolism during endurance exercise. Am J Clin Nutr. 72 (Suppl): 558S-563S.

[9] Hirsch IB, Marker JC, Smith LJ, Spina R, Parvin CA, Cryer PE (1991) Insulin and glucagon in the prevention of hypoglycemia during exercise in humans. Am J Physiol. 260: E695-704.

[10] Sigal RJ, Fisher SF, Halter JB, Vranic M, Marliss EB (1995) The roles of catecholamines in glucoregulation in intense exercise as defined by the islet cell technique. Diabetes.45: 148-156.

[11] Wasserman DH.,Zinman B (1994) Exercise in individuals with IDDM (Technical review) Diabetes Care. 17: 924-937.

[12] Davis SN, Galassetti, Wasserman DH, Tate DH (2000) Effects of antecedent hypoglycemia on subsequent counterregulatory responses to exercise. Diabetes. 49: 7381.

[13] Hernandez JM, Moccia T, Fluckey JD, Ulbrecht JS, Farrell PA (2000) Fluid snacks to avoid late onset post exercise hypoglicemia. Med Sci Sports Exerc. 32: 904-910.

[14] Nakar VA, Downes M, Yu T, Embler E,Wang Y et al. (2008) AMPK and PPARס agonists are exercise mimetics. Cell.134(3): 405-415.

[15] Goodyear LJ (2008) The Exercise Pill - Too Good to Be True? New Engl J Med.359(17):1842-1844.

[16] Fujii N, Aschenbach WG, Musi N, Hirshman MF, Goodyear LJ (2008) Regulation of glucose transport by the AMP-activated protein kinase. ProcNutr Soc. 63: 205210.

[17] Burke LM, Collier GR, Hargreaves M (1993) Muscle glycogen storage after prolonged exercise: the effect of glycaemic index of carbohydrate feedings. J Appl Physiol.75:10191023.

[18] Diabetes UK (2003) The implementation of nutritional advice for people with diabetes. Diab Med.20:786-807.

[19] Nathan DN, Madnek S, Delahanty L (1985) Programming pre-exercise snacks to prevent post-exercise hypoglycaemia in intensively treated insulin-dependent diabetics. Ann Intern Med. 4:483-86.

[20] American Diabetes Association. (2004) Diabetes and Exercise. Diabetes Care. 27(1):s58s62.

[21] Grimm JJ, Ybarra J, Berné C, Muchnick S, Golay A (2004) A new table for prevention of hypoglucemia during physical activity in type 1 diabetic patients. Diabetes Metab.30:465-470.

[22] Murillo S, Brugnara L, Novials A (2010) One year follow-up in a group of halfmarathon runners with type-1 diabetes treated with insulin analogues. J Sports Med Phys Fitness. 50(4):506-10. 
[23] Mc Mahon SK, Ferreira LD, Ratnam N, Davey RJ, Youngs LM, Davis EA, et al. (2007) Glucose requirements to maintain euglycemia after moderate-intensity afternoon exercise in adolescents with type 1 diabetes are increased in a biphasic manner. J Clin Endocrinol Metab.92(3):963-8. 